\title{
Performance analysis of two-user cooperative ARQ protocol over Rayleigh fading channel
}

\author{
Hailin $\mathrm{XiaO}^{1^{*}}$ and $\mathrm{Ju} \mathrm{Ni}^{2}$
}

\begin{abstract}
In this paper, we present a two-user cooperative automatic repeat request (C-ARQ) protocol which combines cooperative diversity at the physical layer and $A R Q$ at the link layer. In this scheme, distributed Alamouti space-time block coding (DASTBC) is applied to achieve cooperative diversity, and ARQ is used to improve the data link layer reliability. To analyze the performance of the proposed protocol, we derive the average frame error rate (FER) and throughput with multiple phase shift keying (MPSK) over a Rayleigh fading channel. Numerical simulation results show that the performance gain of average FER with $A R Q$ retransmission is about $2 \mathrm{~dB}$ more than the case without $A R Q$ retransmission in the same conditions. Moreover, the combined DASTBC and quadrature phase shift keying (QPSK) with $A R Q$ retransmission will lead to an approximate $3 \mathrm{Mbits} / \mathrm{s}$ increase in transmission rate.
\end{abstract}

Keywords: Cooperative communication; Multiple phase shift keying; ARQ; Space-time block coding

\section{Introduction}

Multiple antennas are often used to combat channel fading in wireless communication system [1]. However, implementing multiple antennas at a mobile station is impractical for most wireless applications due to the limited size of the mobile unit [2,3]. Relay-assisted communication is suitable to solve the problem and plays a main role in the future generation cellular systems and ad hoc networks [4]. One form of relayassisted communication is cooperative diversity $[5,6]$. Its principle is to exploit the broadcast nature of wireless transmission and creates a virtual antenna array through cooperating users. Each transmitting node has its own message and is responsible for transmitting the information of its partner(s). Therefore, a virtual antenna array is obtained through the use of the relay's antennas without complicated signal design or adding more antennas into the nodes. In [7], a cooperative diversity scheme between users has been used not only to obtain higher throughput but also to decrease timevarying channel sensitivity. In [8], multiple-source cooperative message has been sent to a common destination, and it is shown to offer full spatial diversity with better

\footnotetext{
* Correspondence: xhl_xiaohailin@163.com

'School of Information and Communication, Guilin University of Electronic Technology, Guilin 541004, Guangxi, China

Full list of author information is available at the end of the article
}

bandwidth efficiency. In [9], differential relay strategies over downlink channel in a two-user cooperative communication system have been proposed, which are able to achieve performance gain in association with the proposed criterion. Recently, various techniques such as distributed space-time coding $[10,11]$ and modulation [12] have been developed to realize cooperative diversity. For achieving a diversity gain, the Alamouti space-time block code (STBC) has been used in cooperative system in a distributed manner [13]. These cooperative diversity schemes have been shown to be able to achieve significant performance gains over the amplifyand-forward and decode-and-forward protocols [14].

Automatic repeat request (ARQ) protocol is commonly used in the communication system to improve the reliability, which requests retransmissions for those packets received in error [15]. Recently, the ARQ protocol has been applied in cooperative diversity system to achieve higher link reliability; it is called cooperative ARQ [16]. Cooperative ARQ (C-ARQ) protocol permits nodes other than the source and the destination to actively help deliver the correct data $[3,17,18]$. In [3], the authors have analyzed the diversity multiplexing trade-off (DMT) for ARQ-based opportunistic cooperative communication over Nakagami fading channels. In [17], the authors have designed the ARQ protocol for two-user cooperative diversity systems in wireless networks. In [18], a cross-layer 


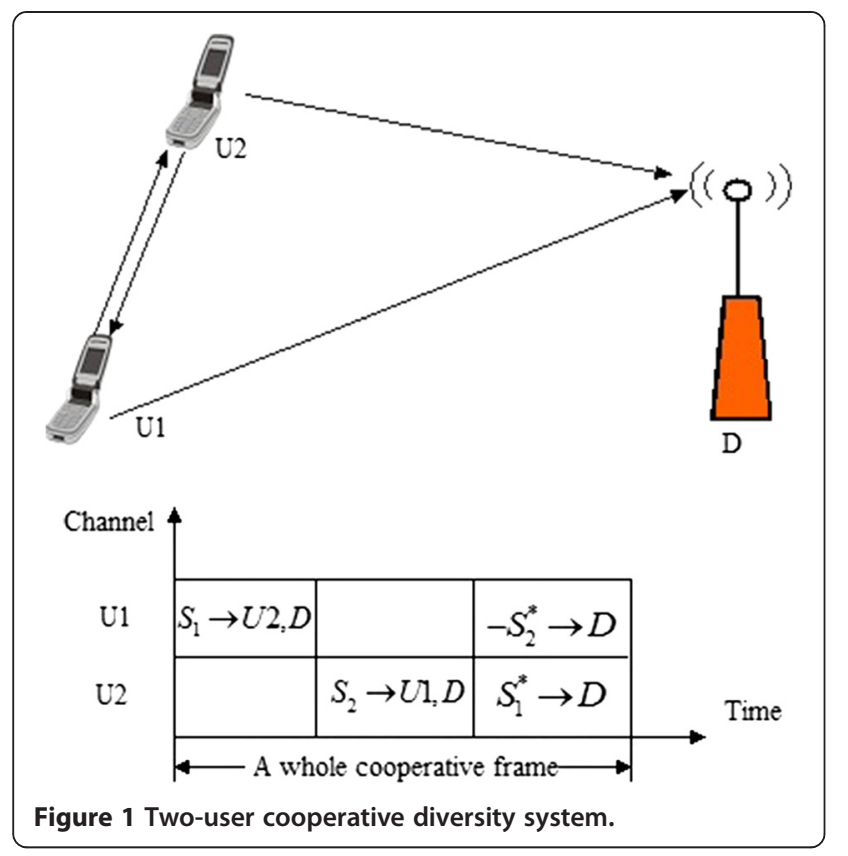

C-ARQ has been studied, and the performance of spectral efficiency over Nakagami fading channel has been evaluated. In [19], a coordinated hybrid automatic repeat request (HARQ) approach has been proposed, which can improve the network outage probability and the users' fairness. Despite that these studies are not relevant to the average frame error rate (FER) and throughput of cooperative ARQ, they can serve as references [20].

In this paper, we present a two-user C-ARQ protocol which combines cooperative diversity at the physical layer and ARQ at the link layer. To analyze the performance of the protocol, we derive the average FER and throughput

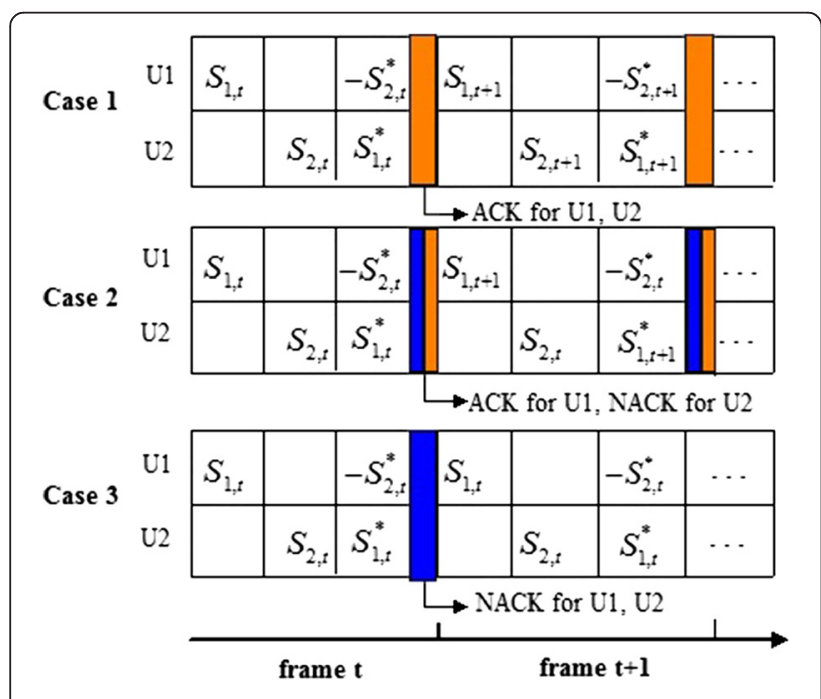

Figure 2 Two-user cooperative ARQ protocol based on [15]. with multiple phase shift keying (MPSK) over a Rayleigh fading channel. Numerical simulation results show that the performance gain of average FER with ARQ retransmission is about $2 \mathrm{~dB}$ more than the case without ARQ retransmission in the same conditions. Moreover, the combined distributed Alamouti space-time block coding (DASTBC) and quadrature phase shift keying (QPSK) with ARQ retransmission will lead to an approximately $3 \mathrm{Mbits} / \mathrm{s}$ increase in transmission rate.

\section{System model}

We consider a cellular system in which two cooperative users transmit their information to the same destination $(D)$ as depicted in Figure 1 [17]. Both users and destination are equipped with a single antenna. The system adopts time division multiple access (TDMA) scheme, and both the half-duplex users share the same frequency band. Thus, to ensure half-duplex operation, we further divide each channel into orthogonal subchannels. Under the above orthogonality constraints, we can now conveniently, and without loss of generality, characterize our channel models using a time-division notation; frequency division counterparts to this model are straightforward due to the symmetry of the channel allocations. Specifically, using system communication, we can provide the powerful benefits of diversity without the need for physical arrays, although at a loss of spectral efficiency due to half-duplex operation and possibly at the cost of additional received hardware.

In this paper, we extend a two-user cooperative diversity system by exploiting the proposed ARQ protocols as follows. A whole cooperative frame is divided into three sub-frames. The first sub-frame is allocated for user 1 (U1) to transmit its data packet. The second sub-frame is assigned to user 2 (U2) to transmit its data packet. The third sub-frame is shared between both users and is used to relay each other's message to the destination. The cyclic redundancy checksum (CRC) is used to facilitate error detection. If one user receives a correct subframe of its partner, it will relay the received information in cooperative sub-frame. Otherwise, the relaying user will do nothing in the third sub-frame. To utilize DASTBC to achieve diversity, U1 relays the packet of U2 with a negative conjugate form. Similarly, U2 transmits a conjugate packet of U1. Then, the destination uses maximum ratio combine (MRC) to receive data packets.

The inter-user channels and the user-destination channels are independent of each other. All channels are assumed to experience block Rayleigh fading, i.e., they are fixed during a frame and change independently in the next frame, where each frame contains a fixed $N_{\mathrm{f}}$ number of bits. Each frame at the physical layer may contain multiple packets from the data link layer. Details of the packet and frame can be found in [21]. If each packet includes $N_{\mathrm{p}}$ symbols, after $M$-ary digital modulation of 
rate $R_{\mathrm{n}}$ bits/symbol, each packet is mapped to a bit block containing $N_{\mathrm{p}} \times R_{\mathrm{n}}$ bits. As in HIPER-LAN/2 and IEEE 802.11a standards [22], each sub-frame is given by:

$$
N_{\mathrm{f}}=N_{\mathrm{c}}+N_{\mathrm{b}} N_{\mathrm{p}} \times R_{\mathrm{n}}
$$

where $N_{\mathrm{c}}$ is the pilot and control parts and $N_{\mathrm{b}}$ is the number of packets per frame which depends on the chosen modulation and coding pair $[23,24]$.

\section{Two-user cooperative ARQ protocol}

For a better description of the two-user cooperative ARQ protocol, we focus on a whole cooperative frame. The destination feeds back CRC checking results of received packets, where an acknowledgement (ACK) or a negative acknowledgement (NACK) message is informed to both users in the ARQ process. It is assumed that the feedback channel is error free and has no latency, and error detection based on CRC is perfect. We consider that the destination has only one ARQ opportunity in a whole cooperative frame. The protocol works as follows and is shown in Figure 2:

1) If the receiver decodes the messages of $U 1$ and $U 2$ correctly. The destination simultaneously transmits an ACK message to U1 and U2. The system will start the next cooperative frame.

2) If the receiver does only decode the messages of U1 correctly. The destination drops the erroneous packets and feeds back two messages in half time slots of feedback duration, an NACK for U2 and ACK for U1. Then, retransmission will be performed by $\mathrm{U} 2$ in the next cooperative frame, while U1 will transmit its next packet in that frame. And vice versa, if the receiver does only decode the messages of U2 correctly, a reciprocity ARQ process is also executed.

3) If the receiver does not decode the messages of $U 1$ and U2 correctly. The destination dumps both erroneous packets and feeds back a NACK message to inform both users to retransmit their incorrect packets in the next frame.

\section{Performance analysis}

In this section, to analyze the performance of the protocol, we derive the average FER and throughput with MPSK over the Rayleigh fading channel.

\subsection{Average frame error rate}

The frame error is determined by the signal-to-noise ratio (SNR). For the flat Rayleigh fading channel, the probability density function (PDF) of instantaneous SNR $\gamma$ is given by:

$$
f_{\gamma}(\gamma)=\frac{1}{\bar{\gamma}} e^{-\frac{\gamma}{\gamma}}
$$

where $\bar{\gamma}$ represents the average SNR. For MRC diversity reception with two independent Rayleigh fading signals, the PDF of the output SNR is given by [25]:

$$
f_{\gamma}(\gamma)=\frac{1}{\bar{\gamma}_{1}-\bar{\gamma}_{2}}\left[\exp \left(-\frac{\gamma}{\bar{\gamma}_{1}}\right)-\exp \left(-\frac{\gamma}{\bar{\gamma}_{2}}\right)\right]
$$

where $\bar{\gamma}_{1}$ and $\bar{\gamma}_{2} \quad\left(\bar{\gamma}_{1} \neq \bar{\gamma}_{2}\right)$ are the average SNR of the two independent diversity branches, respectively. If $\bar{\gamma}_{1}=\bar{\gamma}_{2}$, the PDF of the output of SNR is given by:

$$
f_{\gamma_{n}}(\gamma)=\frac{1}{\bar{\gamma}_{n}} \exp \left(-\frac{\gamma_{n}}{\bar{\gamma}_{n}}\right), n=1,2
$$

where the output SNR can be obtained by the selection combing diversity reception [25].

The average FER $\left(\overline{\mathrm{FER}^{M}}\right)$ with $M$-ary digital modulation can be expressed as:

$$
\overline{\mathrm{FER}^{M}}=\int_{0}^{\infty} P_{e}(b \mid \gamma)^{M} f_{\gamma}(\gamma) d \gamma
$$

where $P_{e}(b \mid \gamma)^{M}$ is the bit error ratio (BER) in nonfading channel and $f_{\gamma}(\gamma)$ is the PDF of the output SNR. For MPSK modulation, the BER is given by [26]:

$$
P_{e}(b \mid \gamma)^{M}=\left\{\begin{array}{cc}
Q(\sqrt{2 \gamma}) & M=2 \\
\approx 2 Q(\sqrt{2 \gamma} \sin (\pi / M)) & M>2
\end{array}\right.
$$

where $Q(\cdot)$ is $Q$ function and can be written as [22]:

$$
Q(x)=\frac{1}{\pi} \int_{\mathrm{x}}^{\pi / 2} \exp \left(-x^{2} / 2 \sin ^{2} \theta\right) d \theta
$$

With (2), (5), and (6), the average FER with MPSK modulation can be calculated as:

$$
\begin{aligned}
\overline{\mathrm{FER}_{n}^{2}} & =\int_{0}^{\infty} \mathrm{P}_{e}(b \mid \gamma)^{M} \frac{1}{\bar{\gamma}} e^{-\frac{\gamma}{\gamma}} d \gamma \\
& =\frac{1}{\pi} \int_{0}^{\frac{\pi}{2}} \int_{0}^{\infty} \exp \left[-\left(\frac{1}{\sin ^{2} \theta}+\frac{1}{\bar{\gamma}_{n}}\right) d \gamma d \theta\right] \\
& =\frac{\bar{\gamma}_{n}}{2}\left(1-\sqrt{\frac{\bar{\gamma}_{n}}{\bar{\gamma}_{n}+1}}\right)(M=2) \\
\overline{\mathrm{FER}_{n}^{M}} & =\int_{0}^{\infty} P_{e}(b \mid \gamma)^{M} \frac{1}{\bar{\gamma}_{n}} e^{-\frac{\gamma}{\gamma_{n}}} d \gamma \\
& =\bar{\gamma}_{n}\left(1-\sqrt{\frac{\sin ^{2}(\pi / M) \bar{\gamma}_{n}}{\sin ^{2}(\pi / M) \bar{\gamma}_{n}+1}}\right)(M>2)
\end{aligned}
$$

where $\bar{\gamma}_{n}$ is the average SNR of the two independent diversity branches, respectively. Substituting (3) and (6) into (5), the average FER with MRC and MPSK modulation are given by: 


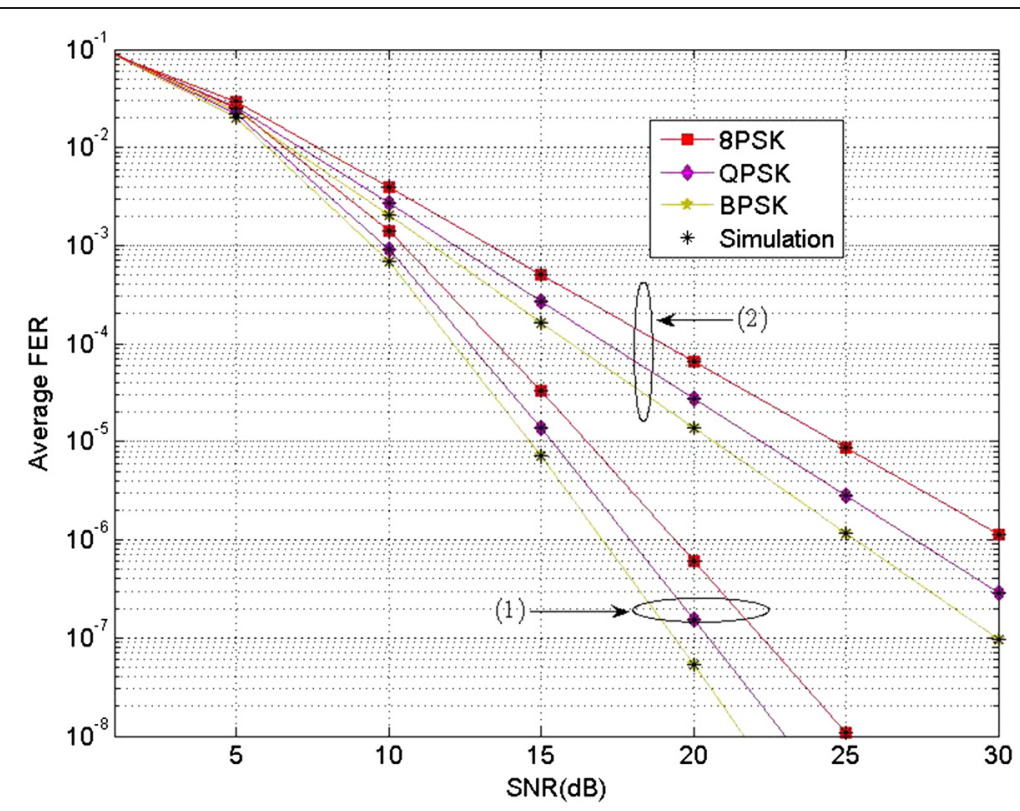

Figure 3 Average FER without ARQ retransmission under $\bar{\gamma}_{1}=2 \bar{\gamma}_{2}(1)$ and $\bar{\gamma}_{1}=3 \bar{\gamma}_{2}$ (2).

$$
\begin{aligned}
\overline{\mathrm{FER}_{\mathrm{MRC}}^{2}} & =\frac{1}{\pi\left(\bar{\gamma}_{1}-\bar{\gamma}_{2}\right)} \int_{0}^{\frac{\pi}{2}}\left(\frac{\bar{\gamma}_{1} \sin ^{2} \theta}{\bar{\gamma}_{1}+\sin ^{2} \theta}-\frac{\bar{\gamma}_{2} \sin ^{2} \theta}{\bar{\gamma}_{2}+\sin ^{2} \theta}\right) d \theta \\
& =\frac{1}{2\left(\bar{\gamma}_{1}-\bar{\gamma}_{2}\right)}\left[\bar{\gamma}_{1}\left(1-\sqrt{\frac{\bar{\gamma}_{1}}{\bar{\gamma}_{1}+1}}\right)-\bar{\gamma}_{2}\left(1-\sqrt{\frac{\bar{\gamma}_{2}}{\bar{\gamma}_{2}+1}}\right)\right](M=2)
\end{aligned}
$$

$$
\begin{aligned}
\overline{\mathrm{FER}_{\mathrm{MRC}}^{M}} & =\frac{1}{\left(\bar{\gamma}_{1}-\bar{\gamma}_{2}\right)}\left[\bar{\gamma}_{1}\left(1-\sqrt{\frac{\sin ^{2}(\pi / M) \bar{\gamma}_{1}}{\sin ^{2}(\pi / M) \bar{\gamma}_{1}+1}}\right)\right. \\
& -\bar{\gamma}_{2}\left(1-\sqrt{\left.\frac{\sin ^{2}(\pi / M) \bar{\gamma}_{2}}{\sin ^{2}(\pi / M) \bar{\gamma}_{2}+1}\right)}\right](M>2)
\end{aligned}
$$

For each whole cooperative frame, we analyze its average FER with ARQ and without ARQ. If there is no ARQ retransmission, two users will not retransmit their respective data packet. The average FER at the destination can be derived as follows:

$$
\begin{aligned}
\overline{\mathrm{FER}^{M}}= & \overline{\mathrm{FER}_{1}^{M}}+\overline{\mathrm{FER}_{2}^{M}}+\left(1-\overline{\mathrm{FER}_{1}^{M}}\right) \\
& \times\left(1-\overline{\mathrm{FER}_{2}^{M}}\right) \overline{\mathrm{FER}_{\mathrm{MRC}}^{M}}
\end{aligned}
$$

According to three cases of the proposed C-ARQ protocol, the average FER at the destination are respectively given by the following:
Case 1: U1 and U2 transmit correct packets.

$$
\begin{aligned}
\overline{\mathrm{FER}^{M}}= & \overline{\mathrm{FER}_{1}^{M}}+\overline{\mathrm{FER}_{2}^{M}}+\left(1-\overline{\mathrm{FER}_{1}^{M}}\right)^{2} \\
& \times\left(1-\overline{\mathrm{FER}_{2}^{M}}\right)^{2} \overline{\mathrm{FER}_{\mathrm{MRC}}^{M}}
\end{aligned}
$$

Case 2 (U1): U1 transmits erroneous packets.

$$
\begin{aligned}
\overline{\mathrm{FER}^{M}}= & \overline{\mathrm{FER}_{1}^{M}}+\overline{\mathrm{FER}_{2}^{M}}+\left(1-\overline{\mathrm{FER}_{1}^{M}}\right)^{2} \\
& \times\left[1-\left(\overline{\mathrm{FER}_{2}^{M}}\right)^{2}\right] \overline{\mathrm{FER}_{\mathrm{MRC}}^{M}}
\end{aligned}
$$

Case 2 (U2): U2 transmits erroneous packets.

$$
\begin{aligned}
\overline{\mathrm{FER}^{M}}= & \overline{\mathrm{FER}_{1}^{M}}+\overline{\mathrm{FER}_{2}^{M}}+\left[1-\left(\overline{\mathrm{FER}_{1}^{M}}\right)^{2}\right] \\
& \times\left(1-\overline{\mathrm{FER}_{2}^{M}}\right)^{2} \overline{\mathrm{FER}_{\mathrm{MRC}}^{M}}
\end{aligned}
$$

Case 3: U1 and U2 transmit erroneous packets.

$$
\begin{aligned}
\overline{\mathrm{FER}^{M}}= & \overline{\mathrm{FER}_{1}^{M}}+\overline{\mathrm{FER}_{2}^{M}}+\left[1-\left(\overline{\mathrm{FER}_{1}^{M}}\right)^{2}\right] \\
& \times\left[1-\left(\overline{\mathrm{FER}_{2}^{M}}\right)^{2}\right] \overline{\mathrm{FER}_{\mathrm{MRC}}^{M}}
\end{aligned}
$$

\subsection{Throughput analysis}

Throughput is defined as the ratio between the number of information bits correctly transmitted by a user and the time that the channel is allocated to that user in a multi-user system $[18,27]$. Hence, the throughput can be calculated as follows:

$$
\text { Throughput }(M)=\frac{P_{\mathrm{f}}}{t} N_{\mathrm{f}}
$$

where $P_{\mathrm{f}}$ is the average FER at the destination. Each frame lasts $t$ seconds. 


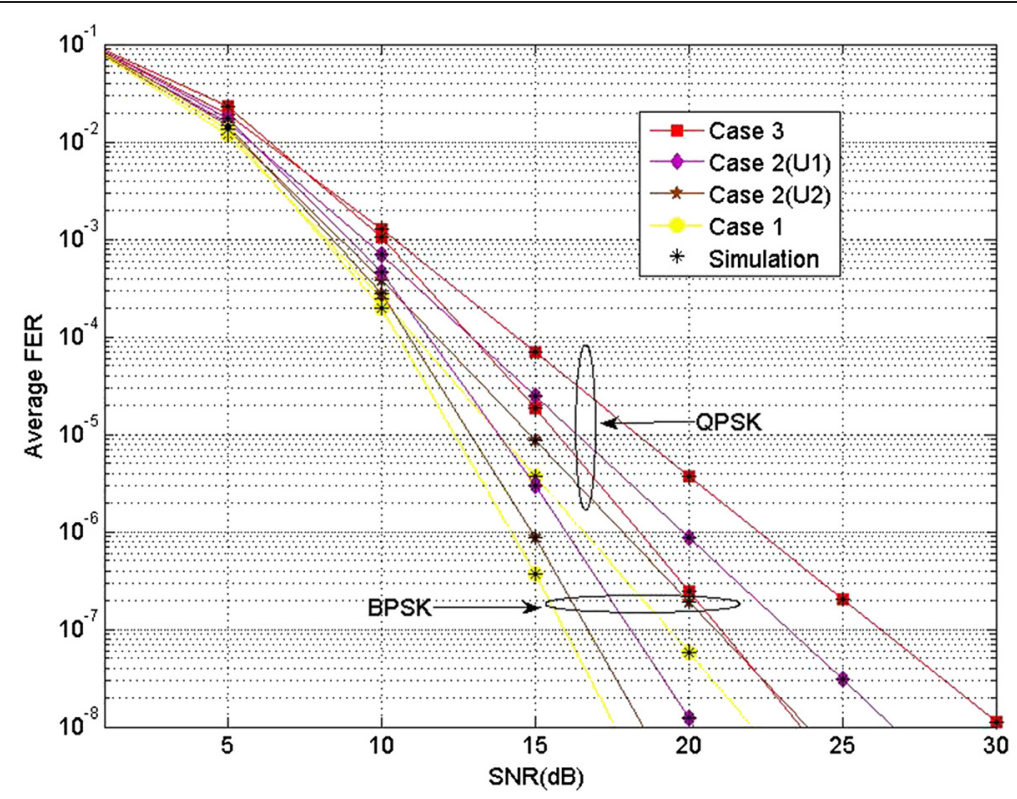

Figure 4 Average FER with ARQ retransmission under $\bar{\gamma}_{1}=2 \bar{\gamma}_{2}$.

\section{Numerical simulation results}

In this section, numerical simulation results are provided to present the average FER performance analysis of the two-user C-ARQ protocol. We set the number of packets $N_{\mathrm{b}}=1,000$. Each packet is set to be 1,000 symbols $\left(N_{\mathrm{p}}=1,000\right)$, and the pilot and control parts are set to be 4 symbols $\left(N_{\mathrm{c}}=4\right)$. Each channel realization is generated as random matrix whose elements are assumed to be circularly symmetric complex Gaussian with zero mean and unity variance. Ten thousand channel matrices are generated with Monte Carlo simulations. For each scenario, 1,000,000 simulation runs are used to obtain each simulated point. The transmitters have normalized bandwidth $W=1 \mathrm{M}$ (it is straightforward to extend the results to the cases with different bandwidths).

Figure 3 illustrates the average FER without ARQ retransmission under $\bar{\gamma}_{1}=2 \bar{\gamma}_{2}$ and $\bar{\gamma}_{1}=3 \bar{\gamma}_{2}$. We consider these cases by fixing one of the users' channels to the destination at a relatively high SNR, i.e., $\bar{\gamma}_{1}=20 \mathrm{~dB}$. We vary the quality of U2's channel to destination. We observe that numerical simulation results are consistent with analytical solutions. It can be seen that the average FER performance degrades as the asymmetric average SNR between the two diversity branches increases. Moreover, with the increase of the modulation order $M$, the average FER performance gets worse.

Figure 4 illustrates the average FER with ARQ retransmission under $\bar{\gamma}_{1}=2 \bar{\gamma}_{2}$. The figure shows that numerical simulation results are consistent with analytical solutions in all cases. As expected, case 3 has a bad average FER performance in the same condition. Comparing Figure 4 with
Figure 3, the performance of average FER with ARQ retransmission is much better than the case without ARQ retransmission, and it is shown that the performance gain is about $2 \mathrm{~dB}$ at an average FER of $10^{-4}$.

Figure 5 shows the throughput with ARQ retransmission under $\overline{\gamma_{1}}=2 \overline{\gamma_{2}}$. It can be seen that the throughput of BPSK modulation is worse than QPSK modulation at the high SNR. The cooperative transmission with BPSK and ARQ retransmission performs best when the SNR is low, because BPSK is more robust to channel fading. Moreover, we observe that ARQ retransmission can achieve sufficient throughput gain. The combined DASTBC and

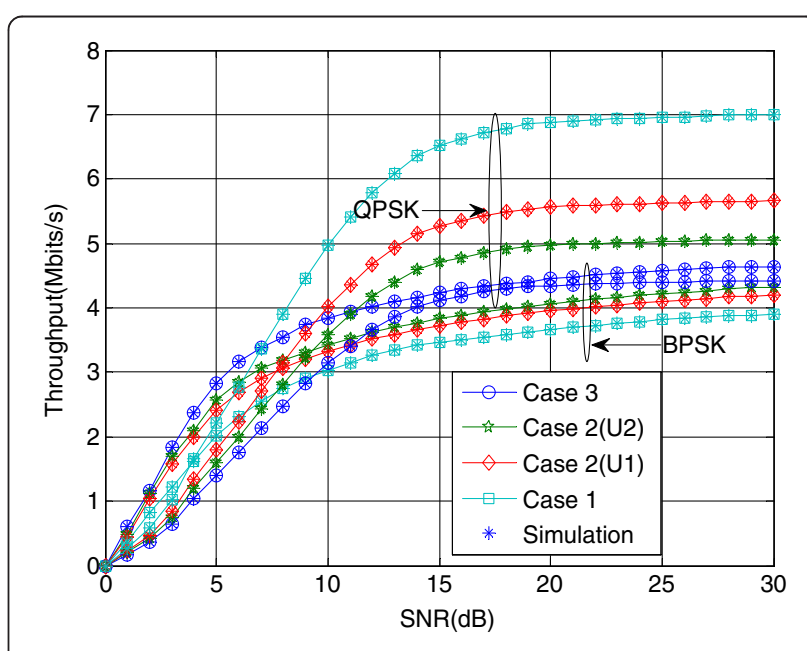

Figure 5 Throughput with ARQ retransmission under $\bar{\gamma}_{1}=2 \bar{\gamma}_{2}$. 
QPSK with ARQ retransmission will lead to an approximately $3 \mathrm{Mbits} / \mathrm{s}$ increase in transmission rate.

\section{Conclusions}

In this paper, we have presented a two-user C-ARQ protocol. To analyze the performance of the protocol, we have derived the average FER and throughput over a Rayleigh fading channel. Numerical simulation results are presented to validate the proposed theoretical analysis. Although we limit two users for simplicity, the proposed scheme can be extended to include more than two users, and the proposed protocol can provide useful basic tools for designing more complicated ARQ protocols. More importantly, our results in this paper offer important analytical method in cooperative communication system.

\section{Competing interests}

The authors declare that they have no competing interests.

\section{Acknowledgements}

The work of H. Xiao and J. Ni is supported by the National Natural Science Foundation of China (Grant No.: 61472094; 61261018; 61362007), Guangxi Natural Science Foundation (Grants No:: 2014GXNSFGA118007 and 2013GXNSFFA019004).

\section{Author details}

${ }^{1}$ School of Information and Communication, Guilin University of Electronic Technology, Guilin 541004, Guangxi, China. 'Library of Guilin University of Electronic Technology, Guilin 541004, Guangxi, China.

Received: 19 August 2014 Accepted: 24 December 2014

Published online: 25 January 2015

References

1. EV Belmega, S Lasaulce, Energy-efficient precoding for multiple-antenna terminals. IEEE Trans. Signal Process. 59(1), 329-340 (2011)

2. H Xiao, S Ouyang, ZP Nie, Design and performance analysis of compact planar inverted-L diversity antenna for handheld terminals. Wireless Pers. Commun. 52(4), 709-717 (2010)

3. JS Harsini, M Zorzi, Effective capacity for multi-rate relay channels with delay constraint exploiting adaptive cooperative diversity. IEEE Trans. Wireless C. 11(9), 3136-3147 (2012)

4. NH Tran, LJ Rodriguez, T Le-Ngocand, HR Bahrami, Precoding and symbol grouping for NAF relaying in BICM systems. IEEE Trans. Vehicular. Technol. 62(6), 2607-2617 (2013)

5. SS Ikki, MH Ahmed, Performance analysis of cooperative diversity with incremental best relay technique over Rayleigh fading channels. IEEE Trans. Commun. 59(8), 2152-2161 (2011)

6. T Liu, L Song, Y Li, Q Huo, B Jiao, Performance analysis of hybrid relay selection in cooperative wireless systems. IEEE Trans. Commun. 61(3), 779-788 (2013)

7. C-H Choi, U-K Kwon, Y-J Kim, G-H Im, Spectral efficient cooperative diversity technique with multi-layered modulation. IEEE Trans. Commun. 58(12), 3480-3490 (2010)

8. H Ding, J Ge, DB da Costa, Z Jiang, A new efficient low complexity scheme for multi-source multi-relay cooperative networks. IEEE Trans. Vehicular. Technol. 60(2), 716-722 (2011)

9. MR Bhatnagar, A Hjørungnes, Differential relay strategies over downlink channel in two-user cooperative communication system. Wireless Pers. Commun. 53(1), 1-14 (2010)

10. J Wu, H Hu, M Uysal, High-rate distributed space-time-frequency coding for wireless cooperative networks. IEEE Trans. Wireless Commun. 10(2), 614-625 (2011)

11. A Nasri, R Schober, M Uysal, Performance and optimization of network-coded cooperative diversity systems. IEEE Trans. Commun. 61(3), 1111-1122 (2013)

12. MR Bhatnagar, Decode-and-forward-based differential modulation for cooperative communication system with unitary and nonunitary constellations. IEEE Trans. Vehicular Technol. 61(1), 152-165 (2012)
13. A Bansal, MR Bhatnagar, A Hjørungnes, Decoding and performance bound of demodulate-and-forward based distributed Alamouti STBC. IEEE Trans. Wireless Commun. 12(2), 702-713 (2013)

14. YF Chen, R Shi, M Long, Performance analysis of amplify-and-forward relaying with correlated links. IEEE Trans. Vehicular Technol. 62(5), 2344-2349 (2013)

15. I Stanojev, O Simeone, U Spagnolini, Y Bar-Ness, RL Pickholtz, Cooperative ARQ via auction-based spectrum leasing. IEEE Trans. Commun. 58(6), 1843-1856 (2010)

16. M Mardani, JS Harsini, F Lahouti, B Eliasi, Link-adaptive and QoS-provisioning cooperative $A R Q$ - applications to relay-assisted land mobile satellite communications. IEEE Trans. Vehicular Technol. 60(7), 3192-3206 (2011)

17. C Zhang, W Wang, G Wei, Design of ARQ protocols for two-user cooperative diversity systems in wireless networks. Comput. Commun. 32(6), 1111-1117 (2009)

18. N Marchenko, C Bettstetter, Cooperative ARQ with relay selection: an analytical framework using semi-Markov processes. IEEE Trans. Vehicular Technol. 63(1), 178-190 (2014)

19. B Makki, T Svensson, T Eriksson, M Alouini, Coordinated hybrid automatic repeat request. IEEE Commun. Lett. 18(11), 1975-1978 (2014)

20. M Behrooz, T Eriksson, T Svensson, On an HARQ-based coordinated multi-point network using dynamic point selection. EURASIP J. Wireless Commun. Netw. 2013(209), 1-11 (2013)

21. Q Liu, S Zhou, GB Giannakis, Cross-layer combining of adaptive modulation and coding with truncated ARQ over wireless links. IEEE Trans. Wireless Commun. 3(5), 1746-1754 (2004)

22. A Doufexi, S Armour, M Butler, A Nix, D Bull, J McGeehan, P Karlsson, A comparison of the HIPERLAN/2 and IEEE 802.11a wireless LAN standards. IEEE Commun. Mag. 40(5), 172-180 (2002)

23. J Wang, OY Wen, S Li, Near optimum pilot and data symbols power allocation for MIMO spatial multiplexing system with zero-forcing receiver. IEEE Signal. Process. Lett. 16(5), 358-361 (2009)

24. X Rui, J Hou, L Zhou, Decode-and-forward with full-duplex relaying. Int. J. Commun. Syst. 25(5), 270-275 (2012)

25. L Fang, G Bi, AC Kot, New method of performance analysis for diversity reception with correlated Rayleigh-fading signals. IEEE Trans. Vehicular. Technol. 49(5), 1807-1812 (2000)

26. JG Proakis, Digital Communications, 4th edn. (McGraw-Hill, New York, 2001)

27. H Zheng, A Lozano, M Haleem, Multiple ARQ process for MIMO systems. EURASIP J. Appl. Signal. Process. 2004(5), 772-782 (2004)

\section{Submit your manuscript to a SpringerOpen ${ }^{\circ}$ journal and benefit from:}

- Convenient online submission

- Rigorous peer review

- Immediate publication on acceptance

- Open access: articles freely available online

- High visibility within the field

- Retaining the copyright to your article

Submit your next manuscript at springeropen.com 